\title{
Plasminogen activators are involved in angiostatin generation in vivo in benign and malignant ovarian tumor cyst fluids
}

\author{
A.A.G. VAN TILBORG ${ }^{1,2}$, F.C.G.J. SWEEP ${ }^{3}$, A.J. GEURTS-MOESPOT ${ }^{3}$, A.M.M. WETZELS ${ }^{1}$, \\ R.M.W. DE WAAL ${ }^{1}$, J.R. WESTPHAL ${ }^{1}$ and L.F.A.G. MASSUGER ${ }^{1}$ \\ Departments of ${ }^{1}$ Obstetrics and Gynaecology, ${ }^{2}$ Pathology, and ${ }^{3}$ Laboratory Medicine, \\ Radboud University Medical Center, Nijmegen, The Netherlands
}

Received November 29, 2013; Accepted January 22, 2014

DOI: $10.3892 /$ ijo.2014.2303

\begin{abstract}
In many tumor types, angiogenesis is the net result of pro- and anti-angiogenic mediators and correlated with metabolic activity, growth, and degree of malignancy. One of the first discovered anti-angiogenic compounds is angiostatin, a proteolytic fragment of plasminogen. The requirements for in vivo angiostatin generation have not yet been determined. We investigated the levels of plasminogen and angiostatin by western blotting and of components of the plasminogen activator complex by ELISA in cyst fluid derived from benign and malignant ovarian tumors. Fluid samples from functional ovarian follicles, dermoid cysts and endometriotic lesions were evaluated separately. When no or minimal amounts of plasminogen were present in the cyst fluids, angiostatin was generally absent as well, irrespective of plasminogen activator concentrations. When plasminogen was present, the degree of conversion of plasminogen to angiostatin was significantly correlated with the level of uPA, and, to a lesser extent, to the tPA level. However, angiostatin was also found in a number of cyst fluid samples with minimal or no plasminogen activators, suggesting the involvement of other angiostatin generating proteases in these samples. Conversely, no angiostatin was observed in a number of cyst fluid samples containing both plasminogen and plasminogen activators. The presence of an inhibitor of the enzymatic activity of uPA and/or tPA, like PAI-1, may explain this finding. Our data show that plasminogen activators are clearly involved in in vivo angiostatin formation in ovarian cysts. Most likely, however, other proteases, as well as inhibitors of plasminogen activators, are involved as well.
\end{abstract}

Correspondence to: Dr Angela van Tilborg, Department of Obstetrics and Gynaecology, Radboud University Nijmegen Medical Centre, Geert Grooteplein-Zuid 10, House post 791, 6525 GA Nijmegen, The Netherlands

E-mail: angela.vantilborg@radboudumc.nl

Key words: angiogenesis, angiostatin, plasminogen activator, ovarian tumor, endometriosis

\section{Introduction}

All tissues are dependent on angiogenesis for their sustained growth. In the case of pathological tissue growth, i.e., in malignant tumors and endometriosis, angiogenesis is also required for the formation of metastases and ectopic endometriotic lesions, respectively $(1,2)$. Angiogenesis is considered to be the result of a net balance between the actions of pro- and antiangiogenic factors (3-5). Pro-angiogenic factors include growth factors such as vascular endothelial growth factor (VEGF) $(6,7)$, proteases (plasminogen activators, matrix metalloproteinases or MMPs; $(8,9)$, integrin adhesion molecules $\left(\alpha_{v} \beta_{3}\right.$, $\left.\alpha_{\mathrm{v}} \beta_{5}\right)(10,11)$, extracellular matrix components (collagens, laminins, proteoglycans) and many others. The discovery of angiostatin, a proteolytic fragment of plasminogen (12), and endostatin (13), a proteolytic fragment of collagen XVIII, have prompted studies on anti-angiogenic factors. Both angiostatin and endostatin have been shown to possess potent antiangiogenic and, as a consequence, antitumor activity in several animal tumor models $(14,15)$.

It has been shown that a combination of a plasminogen activator (PA) such as urokinase- and tissue type PA (uPA, tPA), and a free sulfhydryl donor (FSD), can adequately generate angiostatin in vitro. The plasminogen activator is required for the conversion of plasminogen to the active protease plasmin. Subsequently plasmin, in the presence of an FSD, excises the angiostatin fragment from other plasmin molecules (16). We found in a panel of 75 different cell lines derived from 8 different tumor types, that plasminogen activator expression and the ability to generate angiostatin in vitro were clearly linked (17). Other reports have shown that several species of MMPs $(18,19)$, PSA $(20)$, as well as cathepsin V (21) also play a role in angiostatin generation. The fact that several (combinations of) proteases, that each cleave the plasminogen/plasmin molecule at a different location, are able to generate angiostatin, explains the formation of several different angiostatin species. The angiostatin species described in the original paper by O'Reilly et al (12) consisted of the first 3 kringle domains (out of 5) of plasminogen (K1-3), but also K1-4, K1-4.5 and K5 species have been reported (22). Although each angiostatin species has anti-angiogenic properties (probably by inducing apoptosis in vascular cells), the systemic and/or site-specific occurrence of these different species, as well as 
their (relative) contributions to the (anti-)angiogenic balance is unclear. In addition, little is known about the mechanisms involved in the in vivo generation of angiostatin. Rotenberg et al (23) determined the levels of angiostatin, uPA and MMPs in ascitic and pleural effusions of 21 cancer patients, and found no correlation between the presence of angiostatin and either enzyme.

To address this question in a different patient group, we collected cyst fluids from benign and malignant ovarian tumors, and from functional cysts. In these fluids, we determined the presence of plasminogen and angiostatin semiquantitatively by western blot analysis, and the corresponding levels of uPA and tPA by specific ELISAs. The absence or presence of angiostatin in the cyst fluids was linked to the levels of plasminogen, uPA and tPA.

\section{Materials and methods}

Patients. Patients with a cystic ovarian process scheduled for surgical treatment were included in the cyst fluid collection procedure $(n=124)$. Informed consent was obtained from all the patients. We included samples from ovarian carcinoma $(n=31)$, borderline tumors $(n=17)$, benign mucinous ovarian tumors $(n=27)$, benign serous ovarian tumors $(n=28)$, dermoid cysts $(n=11)$ and endometriotic lesions $(n=10)$. After surgical removal, the tissue was immediately transported to the pathology laboratory where aseptic fine needle aspiration was performed to collect cyst fluid samples. Subsequently, cooled fluid samples were centrifuged at 3,000 x g for $10 \mathrm{~min}$. The supernatant was collected and stored at $-35^{\circ} \mathrm{C}$ until further analyses. Histopathological evaluation was performed by an experienced gynecologic pathologist, and clinicopathologic characteristics were retrieved from the medical records of the patients. For comparison we used follicle fluid collected routinely from functional cysts during an IVF procedure from 24 women.

ELISAs for plasminogen activator components. Determination of UPA and tPA concentration was performed by specific double determinant ELISAs as described previously $(24,25)$.

Purification of plasminogen and angiostatin. Lysine-sepharose slurry $(50 \mu \mathrm{l})$ prepared according to the manufacturer's instructions (Pharmacia, Uppsala, Sweden) was added to $200 \mu \mathrm{l}$ of cyst fluid, and incubated overnight on a roller bank at $4^{\circ} \mathrm{C}$. Lysine-sepharose was washed twice with phosphate buffered saline, and bound material was eluted with $100 \mu \mathrm{l}$ $0.2 \mathrm{M} \varepsilon$-amino-capronic acid.

Western blot analyses for plasminogen and angiostatin. SDS-PAGE and western blot analyses were performed essentially as described previously (17). Briefly, samples of $15 \mu \mathrm{l}$ purified plasminogen/angiostatin plus $15 \mu \mathrm{l}$ sample buffer were run on a $10 \%$ polyacrylamide gel. After electrophoresis, samples were electroblotted onto nitrocellulose membranes (Schleicher \& Schuell, Dassel, Germany). Blots were blocked for $60 \mathrm{~min}$ in blocking solution [PBS/0.05\% Tween-20/2\% block (Roche, Basel, Switzerland)], followed by overnight incubation with polyclonal rabbit anti-human antibody, affinity purified against plasminogen kringle domains $1-3$, diluted in blocking solution. After washing, blots were incubated for two hours with peroxidase-conjugated swine anti-rabbit secondary antibody (Dako, Glostrup, Denmark). After washing, blots were developed by chemiluminescence according to the manufacturer's protocol (Roche). Plasminogen and angiostatin content were assessed semiquantitatively by visually scoring the intensity of the resulting bands as absent (0), low (1), moderate (2) or high (3). In addition, the conversion level of plasminogen to angiostatin (irrespective of the accompanying plasminogen and angiostatin levels) was scored in 5 different categories. Category 1 refers to cyst fluids in which no plasminogen was converted at all, and category 5 to cases of complete conversion of plasminogen to angiostatin (in other words no remaining plasminogen present). A further three categories of partial conversion were defined as: $2,<50 \%$ of plasminogen converted to angiostatin (plasminogen band more intense); 3, $~ 50 \%$ of plasminogen converted (bands comparable in intensity); and $4,>50 \%$ of plasminogen converted (angiostatin band more intense).

Statistical analysis. In samples with no conversion of plasminogen to angiostatin, the $75 \%$ percentile of plasminogen activator levels was 7.0 and $18.5 \mathrm{ng} / \mathrm{ml}$ for uPA and tPA, respectively. We used these values to define an increased level of activator components in the analyses. Univariable logistic regression was used to study the occurrence of the conversion of plasminogen to angiostatin, to the probability of increased plasminogen activator component. The dependent variable was the probability of increased uPA, increased tPA or an increase in both, respectively. The independent variable was conversion of plasminogen to angiostatin, in five categories as described above. The odds ratios with $95 \%$ confidence intervals are presented.

\section{Results}

Plasminogen and angiostatin levels in ovarian tumor cyst fluid and functional cyst fluid samples. Cyst fluid plasminogen and angiostatin levels were assessed semiquantitatively by western blot analysis. Two examples from each source of cyst fluid are shown in Fig. 1. Whereas the molecular weight (MW) of the plasminogen we found in the different samples appeared to be constant, variations were observed in the molecular weights of the different angiostatin species. In most samples at least two different angiostatin bands were observed. This is particularly clear in sample 1 in Fig. 1, showing two bands of $\sim 37$ and $42 \mathrm{kDa}$. In functional cyst samples, two angiostatin bands in the $50 \mathrm{kDa}$ range could be observed. Next to these rather large differences in molecular weight, some more subtle differences were observed as well. The angiostatin bands in the two mucinous benign cyst fluids, for example (samples 5 and 6 in Fig. 1) are within the $37-40 \mathrm{kDa} 3$ kringle range but have clearly somewhat different molecular weights.

A summary of the semiquantitative assessment of the levels of plasminogen and angiostatin in each cyst fluid sample is shown in Fig. 2 (left panel, plasminogen; middle panel, angiostatin). High levels of plasminogen were notably found in the majority of carcinoma derived samples. In the fluid samples derived from other pathological cysts, a large variation in the 


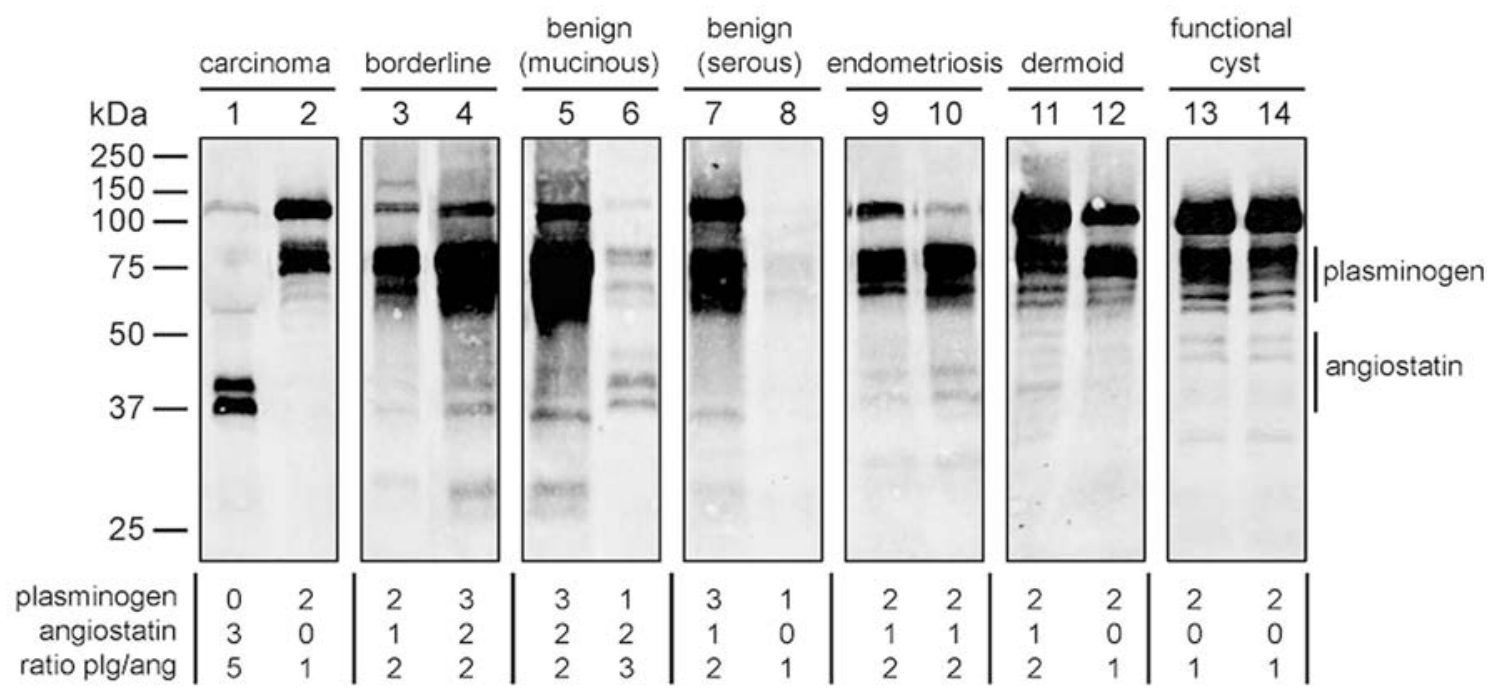

Figure 1. Western blot analysis of plasminogen and angiostatin content of ovarian tumor cyst and functional cyst fluid samples. The origin of the analyzed cyst fluid is shown above each panel; cyst fluid samples derived from 2 different patients per origin are shown. Plasminogen and angiostatin were purified from cyst fluid samples by lysine-sepharose chromatography. A rabbit anti-human plasminogen polyclonal antibody was employed to detect plasminogen ( $92 \mathrm{kDa}$ ) and angiostatin (between 38 and $50 \mathrm{kDa}$ ). MW markers are shown on the left hand side of the panels. Beneath the panels, the semiquantitative scoring, as described in Materials and methods, of plasminogen levels, angiostatin levels and the degree of plasminogen to angiostatin conversion is shown.

Table I. The odds ratio with $95 \%$ confidence interval of enhanced conversion of plasminogen to angiostatin for the probability of increased uPA, increased tPA and increased either uPA or tPA, respectively, using univariable logistic regression.

\begin{tabular}{|c|c|c|c|c|c|c|c|c|c|c|}
\hline \multirow[b]{2}{*}{$\begin{array}{l}\text { Conversion } \\
\text { level }\end{array}$} & \multirow[b]{2}{*}{$\mathrm{n}$} & \multicolumn{3}{|c|}{$\mathrm{uPA}>7.0$} & \multicolumn{3}{|c|}{$\mathrm{tPA}>18.5$} & \multicolumn{3}{|c|}{$(\mathrm{uPA}>7.0)$ or $(\mathrm{tPA}>18.5)$} \\
\hline & & OR & $(95 \% \mathrm{CI})$ & $\mathrm{p}$-value & OR & $(95 \% \mathrm{CI})$ & $\mathrm{p}$-value & OR & $(95 \% \mathrm{CI})$ & $\mathrm{p}$-value \\
\hline 1 & 21 & 1.0 & (ref) & & 1.0 & (ref) & & 1.0 & (ref) & \\
\hline 2 & 56 & 4.0 & $(1.3-12.3)$ & 0.017 & 1.2 & $(0.4-3.8)$ & 0.791 & 1.9 & $(0.7-5.3)$ & 0.093 \\
\hline 3 & 29 & 10.1 & $(2.7-37.5)$ & 0.001 & 3.4 & $(1.0-11.9)$ & 0.052 & 11.6 & $(2.6-50.5)$ & 0.022 \\
\hline 4 & 12 & 3.2 & $(0.7-14.5)$ & 0.132 & 4.5 & $(1.0-20.6)$ & 0.054 & 6.7 & $(1.2-38.25)$ & 0.121 \\
\hline 5 & 5 & 2.1 & $(0.3-16.6)$ & 0.469 & 4.8 & $(0.6-37.4)$ & 0.134 & 5.3 & $(0.5-56.2)$ & 0.289 \\
\hline
\end{tabular}

uPA, urokinase type plasminogen activator; tPA, tissue type plasminiogen activator; OR, odds ratio; CI, confidence interval; plg, plasminogen; ref, reference.

levels of these proteins was observed. In these types of cyst fluids, samples with very large amounts of plasminogen were found, next to samples with no plasminogen at all. Compared to plasminogen, the observed level of angiostatin was generally lower and there were more samples with no angiostatin protein at all. The highest percentages of samples with moderate or high amounts of angiostatin were derived from carcinoma, borderline, and, remarkably, endometriosis cysts.

In contrast to the samples derived from pathological cysts, the functional cyst samples all presented with a rather consistent pattern of moderate amounts of plasminogen and only small amounts (if any) angiostatin. With the exception of the instances mentioned above, no further obvious correlations between source of the cyst fluid, and the presence and/or quantity of either plasminogen or angiostatin were observed.

In the right panel of Fig. 2, the degree of plasminogen to angiostatin conversion is shown, divided in different catego- ries. Categories were based on the ratio between plasminogen and angiostatin content. Category 1 contained those samples where sufficient amounts of plasminogen were present with no conversion to angiostatin whatsoever. Category 5 contained those samples where plasminogen was completely absent due to conversion to angiostatin. These two extremes where rather uncommon, as in many samples both plasminogen as angiostatin was observed, indicating a partial conversion of plasminogen to angiostatin. Category 3 contained those samples where the levels of plasminogen and angiostatin were approximately equal, while in category 2 the conversion to angiostatin was low and in category 4 this was high. The last category contained samples where both plasminogen and angiostatin were not present.

Plasminogen activators in tumor cyst fluid and functional cyst fluid samples. As plasminogen activators are in vitro 


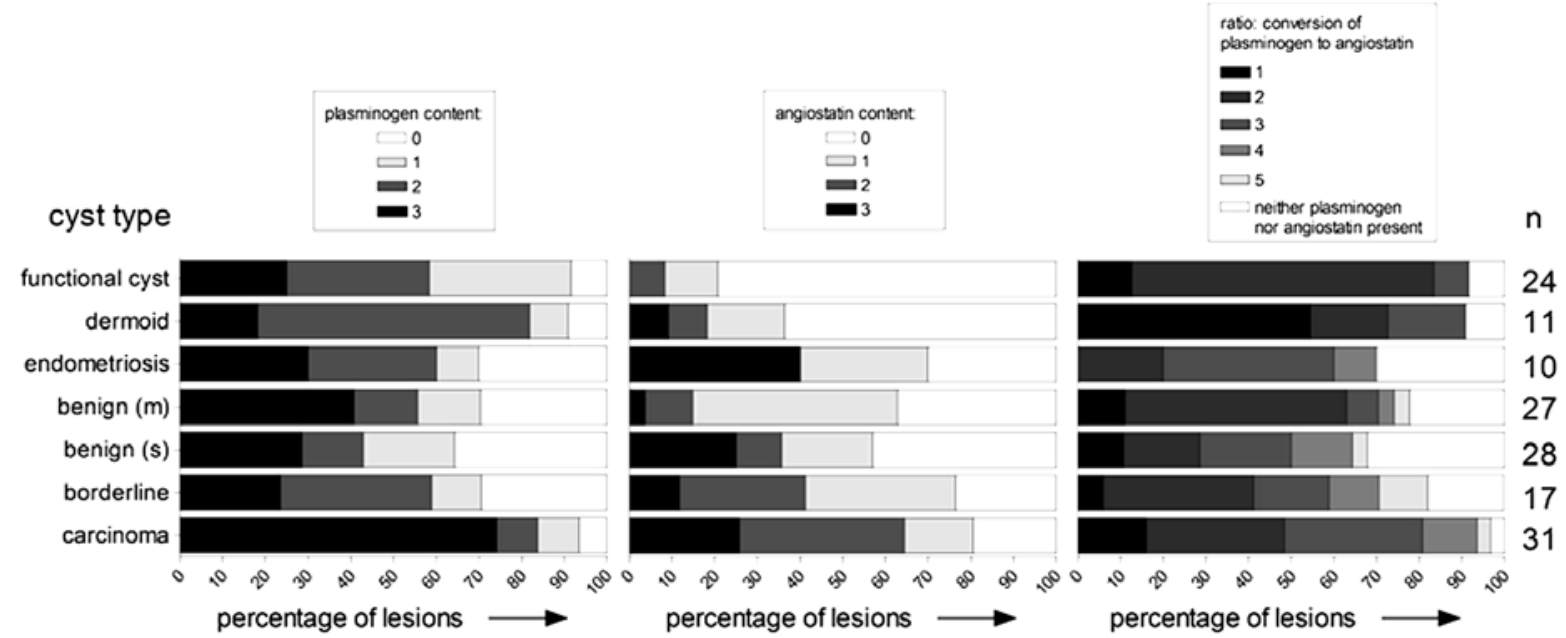

Figure 2. Summary of plasminogen levels (left panel), angiostatin levels (middle panel), both varying from absent ( 0 , white) to high ( 3 , black) and the degree of plasminogen to angiostatin conversion (right panel, on a scale from 1 , little conversion, to 5 , complete conversion) in different types of cyst fluids. The number of samples per group is given on the right side of the figure. Levels and degree of conversion were scored semiquantitatively as described in Materials and methods.

potent mediators of plasminogen to angiostatin conversion, we assessed uPA and tPA levels in the cyst fluid samples to study whether the same mechanism is involved in in vivo angiostatin generation. In Fig. 3, uPA (left panel) and tPA levels (right panel) in the different types of cyst fluid are shown. All cyst fluid groups with the exception of endometriosis (see below) contained a number of samples with no detectable uPA. Median uPA levels tended to be lower in benign lesions. A notable exception was the endometriosis samples, that all contained uPA, some to a very high level. In fact, the mean uPA level in this group was by far the highest and significantly different from all other groups (one-way ANOVA, $\mathrm{p}<0.001$ ). This pattern was not observed for tPA, as this enzyme showed highest median levels in serous benign tumors cyst fluid, followed by borderline tumors and endometriosis. Both uPA and tPA were low in dermoid tumors and functional cysts. The tPA levels in the endometriosis group were not strikingly different from the other cyst fluid groups.

Plasminogen activator levels are associated with the conversion of plasminogen to angiostatin. Fig. 4 shows the levels of uPA (left panel) and tPA (right panel) in relation to the conversion level of plasminogen to angiostatin. Samples that contained neither plasminogen nor angiostatin were excluded from this plot, as these are not informative.

Mean uPA levels were clearly lowest in the samples in which no conversion of plasminogen to angiostatin occurred (category 1). In samples with a low to moderate levels of conversion (categories 2 and 3), uPA levels were significantly higher (t-test, $\mathrm{p}=0.046$ and 0.003 , respectively). With increasing levels of plasminogen to angiostatin conversion (category 4), the mean uPA declined again. Remarkably, in the small group $(n=5)$ of samples with a complete conversion of plasminogen to angiostatin, uPA levels were comparable to those in the group of samples with no conversion at all.

The differences in tPA content between the categories with different levels of plasminogen to angiostatin conversion were less obvious and not significantly different, although the median tPA level increased with increasing conversion levels.

As it is possible that both uPA and tPA are involved in angiostatin generation, and that insufficient levels of one enzyme are compensated for by a sufficient level of the other, we also plotted UPA against tPA concentration for each group of plasminogen to angiostatin conversion (Fig. 5). As in Fig. 4, samples that contained neither plasminogen nor angiostatin were left out of the plot. In 12 of the 21 fluid samples in which no conversion occurred (category 1; Fig. 5 left panel), uPA as well as tPA was $<75 \%$ percentile in this group. In 4 samples, only the tPA concentration $(\leq 78 \mathrm{ng} / \mathrm{ml})$ was increased, while in another 4 only the uPA concentration was increased ( $\leq 16.2 \mathrm{ng}$ / $\mathrm{ml})$. In one sample, both tPA and uPA plasminogen activators (40 and $22 \mathrm{ng} / \mathrm{ml}$, respectively) were increased. Apparently, the presence of plasminogen and uPA and/or tPA is not always sufficient to convert plasminogen to angiostatin.

In categories 2-5 samples, where at least some degree of plasminogen to angiostatin conversion was observed (Fig. 5 right panel), $40 \%$ of samples with a low conversion level (category 2) also showed low uPA and tPA levels (below dotted lines; lower left quadrant of Fig. 5 right panel). Twenty-three percent of category 2 samples, however, presented with high uPA and tPA levels (upper right quadrant), again indicating that the combination of plasminogen and a plasminogen activator is not always sufficient for angiostatin generation. Samples displaying an intermediate, high or complete conversion of plasminogen to angiostatin (categories 3-5), were found predominantly in the upper quadrants, indicating high uPA and/or tPA levels. The probability of increased uPA and/or tPA levels given a certain conversion level is given in Table I. A considerable amount of samples was found in the upper left quadrant (only high tPA levels, 22\%) or lower right quadrant (only high uPA levels, $33 \%$ ). Interestingly, a small minority (five samples, including one with a complete conversion of plasminogen to angiostatin) was positioned in the lower left quadrant, suggesting that in these samples neither UPA nor tPA was involved in angiostatin 


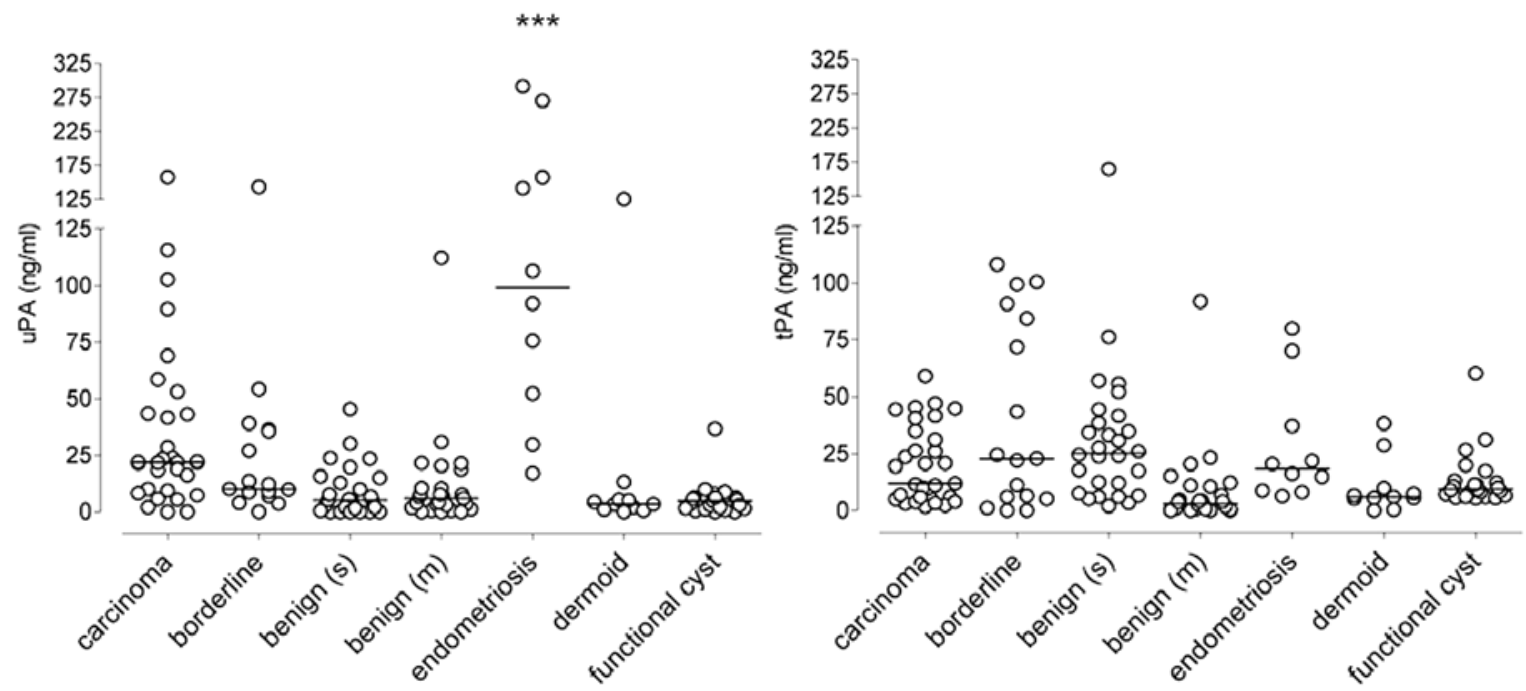

Figure 3. uPA (left panel) and tPA (right panel) levels in different types of cyst fluids. Median levels are shown as horizontal bars.
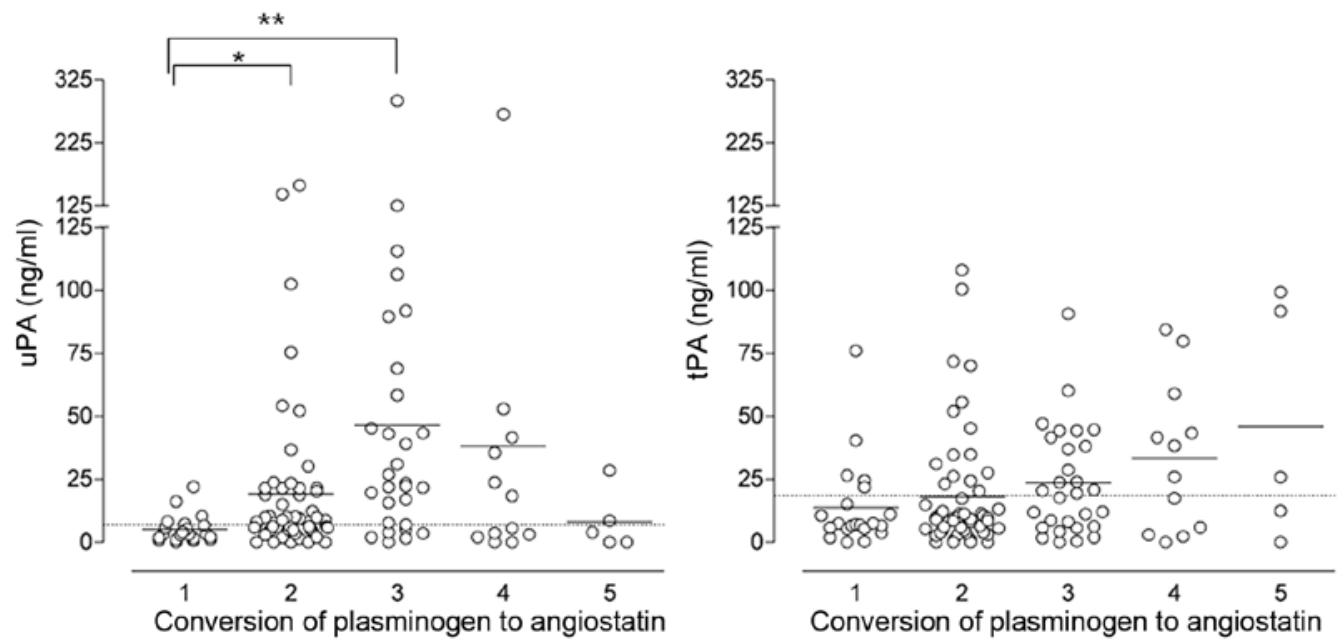

Figure 4. Degree of plasminogen to angiostatin conversion, plotted against uPA (left panel) and tPA (right panel) concentration. Horizontal bars represent mean levels. Dotted lines represent the $75 \%$ percentile of uPA or tPA in category 1 (samples without conversion of plasminogen to angiostatin).

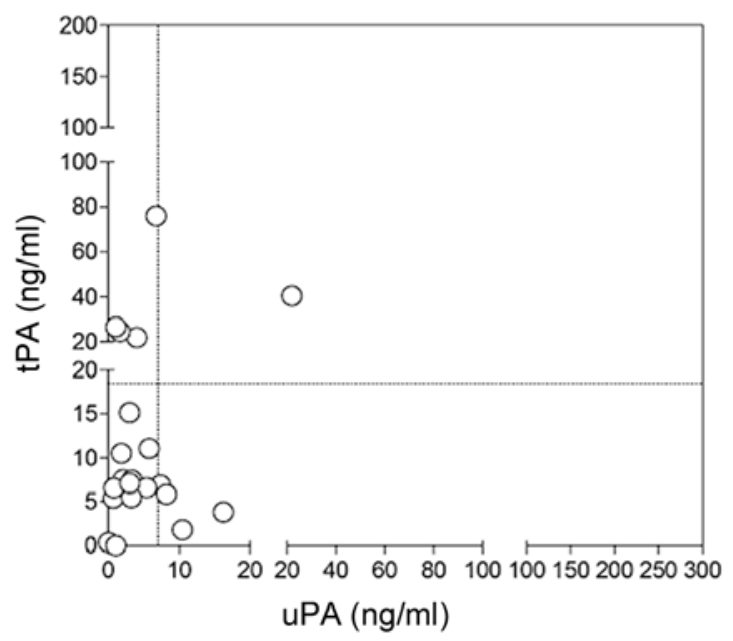

Conversion level $\bigcirc 1$

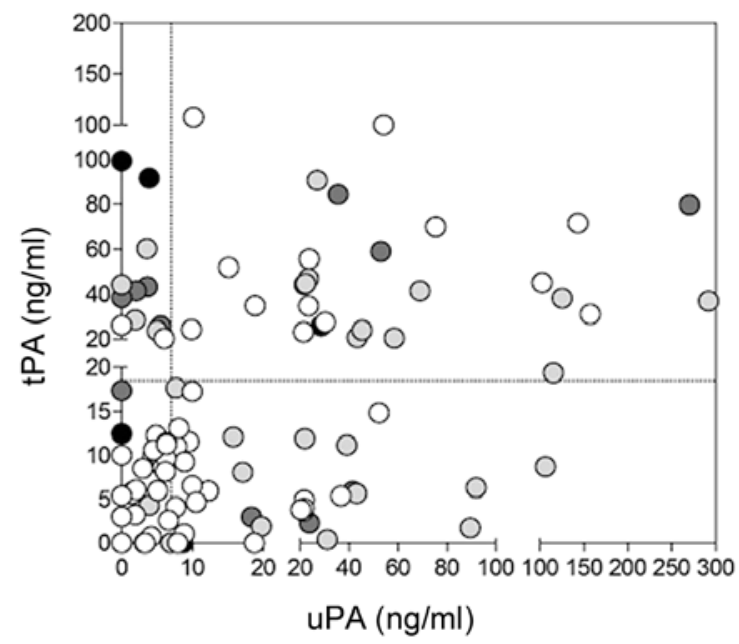

Conversion level $\bigcirc 2 \bigcirc 3 \bigcirc 4 \bullet 5$

Figure 5. Degree of plasminogen to angiostatin conversion plotted against uPA as well as tPA concentration. Left panel, no conversion of plasminogen to angiostatin. Right panel, different levels of plasminogen to angiostatin conversion. Dotted lines represent the 75\% percentiles of uPA and tPA in category 1 (samples without conversion of plasminogen to angiostatin). 
generation. When analyzing our data for each source of cyst fluid separately, we did not find any tumor-specific angiostatin generating pathways (data not shown).

\section{Discussion}

Angiogenesis is a prerequisite for (pathological) tissue growth and the formation of metastatic or ectopic tissue. Angiogenesis is the net result of the balance between of pro- and antiangiogenic molecules. Angiostatin, a proteolytic fragment of plasminogen with potent anti-angiogenic properties, has been extensively studied with regard to the molecules involved in excising the different angiostatin fragments from its parent molecule. Circulating angiostatin has been demonstrated in tumor-bearing as well as control mice, and also in human cancer patients and healthy subjects.

In our angiostatin containing samples we usually observed at least two angiostatin bands, that differed $\sim 4 \mathrm{kDa}$ in molecular weight. These tandem bands were either found in the $37-40 \mathrm{kDa}$ range, or in the 48-52 $\mathrm{kDa}$ range. As the molecular weight of one plasminogen kringle domain is $\sim 12 \mathrm{kDa}$, the two sets of tandem bands may represent angiostatin species containing 3 or 4 kringle domains, respectively. As reported previously, both the 3 kringle and 4 kringle angiostatin species possess anti-angiogenic properties. We also observed angiostatin species in the $37-40 \mathrm{kDa}$ range that were only slightly (1-2 kDa, or even less) different from each other with regard to their molecular weight. Whether these bands represent different glycosylation forms of the same proteolytic fragment, or proteolytic fragments of different sizes, is unknown. Interestingly, the angiostatin species with a molecular weight between 48 and $52 \mathrm{kDa}$ were observed exclusively in the functional cyst samples, whereas the $37-40 \mathrm{kDa}$ species were only found in the pathological samples. This finding suggests that angiostatin generation is different under physiological conditions compared to pathological conditions. We could, however, not detect any differences between the levels of uPA or tPA on one hand, and the molecular weight form of the angiostatin that was generated on the other.

Thus far, the mechanisms involved in physiological as well as pathological angiostatin generation in vivo, have not been elucidated. Rotenberg et al (23) have studied angiostatin levels in blood, and in ascitic and pleural effusions from 21 patients with malignant disease. No link between angiostatin levels, and concentrations of either MMPs or plasminogen activators could be established. Murthi et al (26) analyzed plasminogen levels and UPA activity in tissue extracts and in the urine of patients with normal, benign, borderline, and invasive serous tumors and suggested that proteolytic activity of the plasminogen activation cascade increases in serous epithelial ovarian carcinoma in combination with a decrease in plasminogen and angiostatin levels. Drenberg et al (27) assayed angiostatin levels in plasma and urine by ELISA in normal samples, benign gynecological disease, primary peritoneal cancer and epithelial ovarian cancer. Angiostatin was elevated in urine of ovarian cancer patients regardless of tumor grade, stage, size, histological subtype, creatinine levels, menopausal status, or patient age.

In our study, we show a clear correlation between the concentration of UPA, and angiostatin content in cyst fluids derived from malignant and benign ovarian disease, endometri- otic lesions, and functional cysts. We also identified, however, a number of samples containing angiostatin but no uPA or tPA, and, on the other hand, plasminogen containing samples with substantial levels of uPA, tPA or both, but no angiostatin. In the first group of samples other proteases, for instance members from the MMP family may have been involved in excising the angiostatin moiety from the plasminogen parent molecule. In the second group of samples, molecules such as plasminogen activator inhibitor-1 (PAI-1) may have modulated the enzymatic activity of the plasminogen activator, thus preventing angiostatin generation.

Our data are in accordance with a previous report in which we analyzed the angiostatin generating capacity of a large number of tumor cell lines (17). In this study we found a strong correlation between UPA and tPA production and angiostatin generation, but we also observed angiostatin generation by a number of uPA/tPA negative cell lines.

Our results may explain the dual role that plasminogen activators appear to play in tumor progression. These proteases have been described to be positively- as well as negativelycorrelated with the degree of malignancy of several tumor types, e.g., breast cancer (28-32). The role of plasminogen activators in in vivo angiostatin generation may lead to a shift in the angiogenic balance towards the inhibitory side, resulting in a less malignant tumor and, consequently, in a negative correlation between plasminogen activator expression and tumor malignancy and/or progression.

Using angiostatin as a (potential) treatment, is hampered by problems producing sufficient amounts of active angiostatin, and subsequent effective administration of the compound. In parallel studies $(33,34)$ we have attempted to bypass these problems by trying to stimulate the endogenous in vivo angiostatin production. In a human melanoma xenograft model, administration of a combination of tPA and a free sulfhydryl donor to tumor bearing mice resulted in increased levels of circulating angiostatin, and in an inhibition of tumor growth. Further elucidation of the mechanism(s) involved in in vivo angiostatin production, may eventually lead to the development of alternative anti-angiogenic therapeutic approaches aimed at increasing the circulating levels of angiostatin.

In conclusion, our data show that plasminogen activators are clearly involved in angiostatin formation in vivo in ovarian cysts. Most likely, however, other proteases, as well as inhibitors of plasminogen activators, are involved as well.

\section{References}

1. Folkman J: Seminars in Medicine of the Beth Israel Hospital, Boston. Clinical applications of research on angiogenesis. NEngl J Med 333: 1757-1763, 1995.

2. Folkman J: Angiogenesis in cancer, vascular, rheumatoid and other disease. Nat Med 1: 27-31, 1995.

3. Carmeliet P and Collen D: Molecular analysis of blood vessel formation and disease. Am J Physiol 273: H2091-H2104, 1997.

4. Iruela-Arispe ML and Dvorak HF: Angiogenesis: a dynamic balance of stimulators and inhibitors. Thromb Haemost 78: 672-677, 1997.

5. Talks KL and Harris AL: Current status of antiangiogenic factors. Br J Haematol 109: 477-489, 2000.

6. Bicknell R and Harris AL: Mechanisms and therapeutic implications of angiogenesis. Curr Opin Oncol 8: 60-65, 1996.

7. Liekens S, De Clercq E and Neyts J: Angiogenesis: regulators and clinical applications. Biochem Pharmacol 61: 253-270, 2001. 
8. Hofmann UB, Westphal JR, Van Muijen GN and Ruiter DJ: Matrix metalloproteinases in human melanoma. J Invest Dermatol 115: 337-344, 2000.

9. Pozzi A, Moberg PE, Miles LA, Wagner S, Soloway P and Gardner HA: Elevated matrix metalloprotease and angiostatin levels in integrin alpha 1 knockout mice cause reduced tumor vascularization. Proc Natl Acad Sci USA 97: 2202-2207, 2000.

10. Friedlander M, Brooks PC, Shaffer RW, Kincaid CM, Varner JA and Cheresh DA: Definition of two angiogenic pathways by distinct alpha v integrins. Science 270: 1500-1502, 1995.

11. Brooks PC, Clark RA and Cheresh DA: Requirement of vascular integrin alpha $v$ beta 3 for angiogenesis. Science 264: 569-571, 1994.

12. O'Reilly MS, Holmgren L, Shing Y, et al: Angiostatin: a novel angiogenesis inhibitor that mediates the suppression of metastases by a Lewis lung carcinoma. Cell 79: 315-328, 1994.

13. O'Reilly MS, Boehm T, Shing Y, et al: Endostatin: an endogenous inhibitor of angiogenesis and tumor growth. Cell 88: 277-285, 1997.

14. Holmgren L, O'Reilly MS and Folkman J: Dormancy of micrometastases: balanced proliferation and apoptosis in the presence of angiogenesis suppression. Nat Med 1: 149-153, 1995.

15. O'Reilly MS, Holmgren L, Chen C and Folkman J: Angiostatin induces and sustains dormancy of human primary tumors in mice. Nat Med 2: 689-692, 1996

16. Gately S, Twardowski P, Stack MS, et al: The mechanism of cancer-mediated conversion of plasminogen to the angiogenesis inhibitor angiostatin. Proc Natl Acad Sci USA 94: 10868-10872, 1997.

17. Westphal JR, Van't Hullenaar R, Geurts-Moespot A, et al Angiostatin generation by human tumor cell lines: involvement of plasminogen activators. Int J Cancer 86: 760-767, 2000.

18. Cornelius LA, Nehring LC, Harding E, et al: Matrix metalloproteinases generate angiostatin: effects on neovascularization. J Immunol 161: 6845-6852, 1998.

19. Dong Z, Kumar R, Yang X and Fidler IJ: Macrophage-derived metalloelastase is responsible for the generation of angiostatin in Lewis lung carcinoma. Cell 88: 801-810, 1997.

20. Heidtmann HH, Nettelbeck DM, Mingels A, Jager R, Welker HG and Kontermann RE: Generation of angiostatin-like fragments from plasminogen by prostate-specific antigen. Br J Cancer 81: 1269-1273, 1999

21. Puzer L, Barros NM, Paschoalin T, et al: Cathepsin V, but not cathepsins L, B and K, may release angiostatin-like fragments from plasminogen. Biol Chem 389: 195-200, 2008

22. Soff GA: Angiostatin and angiostatin-related proteins. Cancer Metastasis Rev 19: 97-107, 2000

23. Rotenberg RG, Rozas NS, Guerri L, et al: Elevated levels of angiostatin in effusions from patients with malignant disease. Oncol Rep 11: 523-528, 2004.
24. Grebenschikov N, Geurts-Moespot A, De Witte H, et al: A sensitive and robust assay for urokinase and tissue-type plasminogen activators (uPA and tPA) and their inhibitor type I (PAI-1) in breast tumor cytosols. Int J Biol Markers 12: 6-14, 1997.

25. Span PN, Grebenchtchikov N, Geurts-Moespot J, Westphal JR, Lucassen AM and Sweep CG: EORTC Receptor and Biomarker Study Group Report: a sandwich enzyme-linked immunosorbent assay for vascular endothelial growth factor in blood and tumor tissue extracts. Int J Biol Markers 15: 184-191, 2000.

26. Murthi P, Barker G, Nowell CJ, et al: Plasminogen fragmentation and increased production of extracellular matrix-degrading proteinases are associated with serous epithelial ovarian cancer progression. Gynecol Oncol 92: 80-88, 2004.

27. Drenberg CD, Saunders BO, Wilbanks GD, et al: Urinary angiostatin levels are elevated in patients with epithelial ovarian cancer. Gynecol Oncol 117: 117-124, 2010.

28. de Witte JH, Sweep CG, Klijn JG, et al: Prognostic value of tissue-type plasminogen activator (tPA) and its complex with the type-1 inhibitor (PAI-1) in breast cancer. Br J Cancer 80: 286-294, 1999.

29. de Witte JH, Sweep CG, Klijn JG, et al: Prognostic impact of urokinase-type plasminogen activator (uPA) and its inhibitor (PAI-1) in cytosols and pellet extracts derived from 892 breast cancer patients. Br J Cancer 79: 1190-1198, 1999.

30. Look MP, van Putten WL, Duffy MJ, et al: Pooled analysis of prognostic impact of urokinase-type plasminogen activator and its inhibitor PAI-1 in 8377 breast cancer patients. J Natl Cancer Inst 94: 116-128, 2002.

31. Manders P, Tjan-Heijnen VC, Span PN, et al: Predictive impact of urokinase-type plasminogen activator: plasminogen activator inhibitor type-1 complex on the efficacy of adjuvant systemic therapy in primary breast cancer. Cancer Res 64: 659-664, 2004.

32. Manders P, Tjan-Heijnen VC, Span PN, et al: The complex between urokinase-type plasminogen activator (uPA) and its type-1 inhibitor (PAI-I) independently predicts response to first-line endocrine therapy in advanced breast cancer. Thromb Haemost 91: 514-521, 2004

33. de Groot-Besseling RR, Ruers TJ, Lamers-Elemans IL, Maass CN, de Waal RM and Westphal JR: Angiostatin generating capacity and anti-tumour effects of D-penicillamine and plasminogen activators. BMC Cancer 6: 149, 2006.

34. de Groot-Besseling RR, Ruers TJ, van Kraats AA, et al: Antitumor activity of a combination of plasminogen activator and captopril in a human melanoma xenograft model. Int J Cancer 112: 329-334, 2004. 